\title{
Disfunción renal aguda en pacientes pediátricos que se someten a circulación extracorpórea
}

Alatorre-Orenday Christian Jonathan*, Blancas-Zugarazo Sugela Susana**, Barajas-Salcedo Gerardo***

\begin{tabular}{|c|c|}
\hline 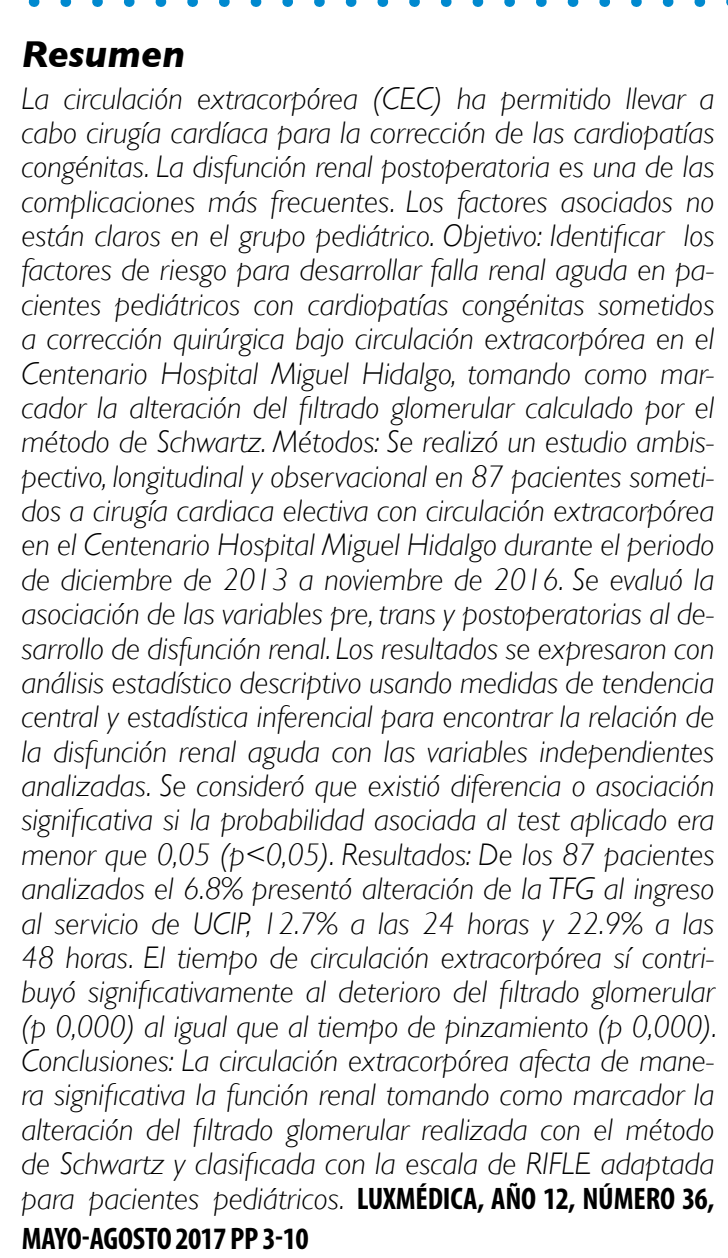 & 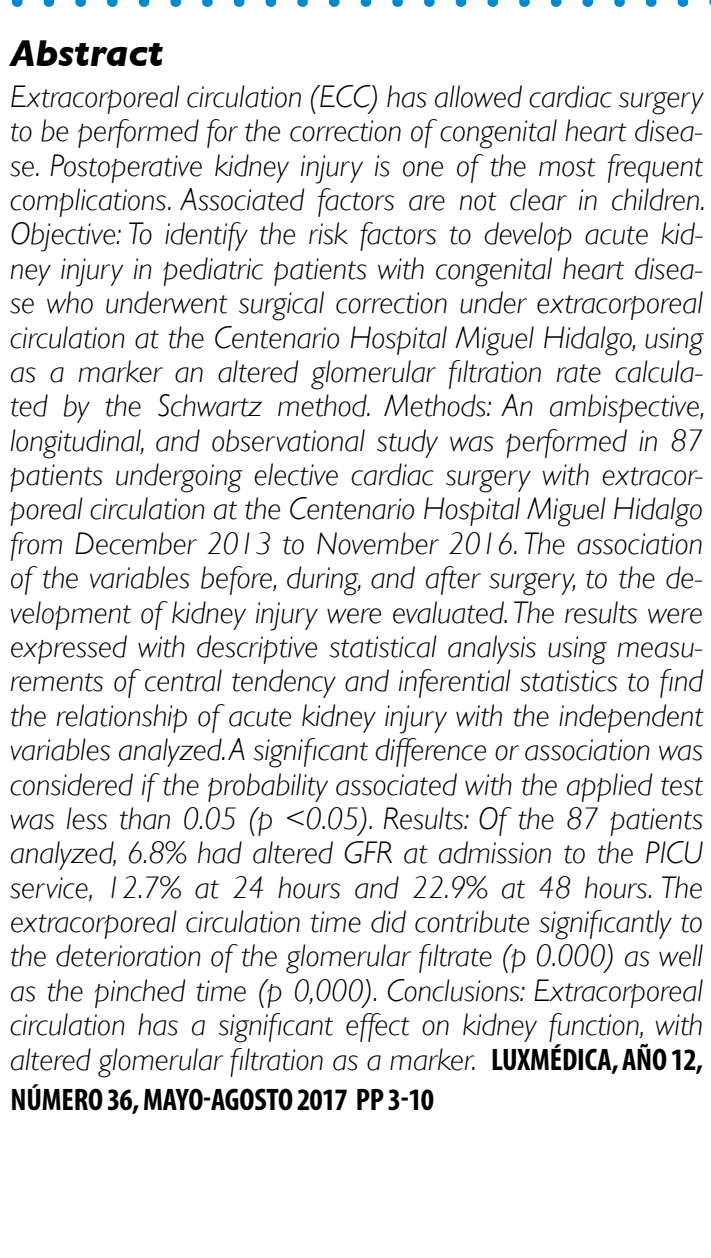 \\
\hline Palabras clave: cardiopatías con & $\begin{array}{l}\text { genital cardiopathies, extracorporeal circu- } \\
\text { jury }\end{array}$ \\
\hline
\end{tabular}

* Médico Pediatra, egresado de la especialidad de Pediatría del Centenario Hospital Miguel Hidalgo

** Profesora investigadora, Cátedra CONACyT, del Centro de Ciencias de la Salud de la Universidad Autónoma de Aguascalientes

*** Médico Pediatra,IIntegrante del Núcleo Académico Básico de la especialidad de Pediatría del Centenario Hospital Miguel Hidalgo

Fecha de recibido: 12 de junio 2017

Fecha de aceptación: 30 junio 2017

Correspondencia: Dr Gerardo Barajas Salcedo, Centenario Hospital Miguel Hidalgo Calle Galeana Sur Número 465 Colonia Obraje. Código Postal 20230. Aguascalientes, Ags, México. Teléfono (01) 4499946720 y 9946726. Correo electrónico gerardobar2006@hotmail.com 


\section{Introducción}

Las cardiopatías congénitas constituyen el grupo más importante de las malformaciones congénitas, con una incidencia de 6 a 8 por cada 1,000 nacidos vivos. Aproximadamente una cuarta parte de estos niños tienen cardiopatías congénitas críticas que requieren cirugía o cateterismo terapéutico durante el primer año de vida. Contribuyen con el $3 \%$ de la mortalidad infantil y el $46 \%$ de las muertes por malformaciones congénitas. En México se estima que nacen entre 12 y 16 mil niños con cardiopatías congénitas cada año y se consideran la primera causa de hospitalización en recién nacidos con malformaciones congénitas. ${ }^{1,2}$ De acuerdo con el reporte del Instituto Nacional de Estadística y Geografía (INEGI) 2013, representan la segunda causa de muerte en niños mexicanos menores de cinco años y en el año 2015 el mismo INEGI reportó las malformaciones congénitas del sistema circulatorio como la cuarta causa de muerte en menores de 1 año..$^{1-3}$ El Centenario Hospital Miguel Hidalgo, es un centro de referencia regional que ofrece la corrección de las cardiopatías congénitas. En los últimos tres años el $30.2 \%$ de los ingresos al servicio de terapia intensiva pediátrica correspondieron a pacientes sometidos a cirugía cardiovascular. ${ }^{4}$ El tratamiento de las cardiopatías congénitas ha mejorado en los últimos años, gracias al desarrollo en las técnicas, diagnósticas y terapéuticas. Las cardiopatías congénitas tienen como características su gran variabilidad de presentación, desde un punto de vista tanto anatómico como fisiopatológico, que condicionan una complejidad en la exactitud diagnóstica, con variabilidad de tratamientos. Hasta la década de los cincuenta del siglo XX la mayoría de las cardiopatías congénitas eran casi curiosidades anatómicas y sólo era posible realizar tratamientos paliativos. La aparición del oxigenador de membrana supuso un cambio radical en la cirugía, condicionó el poder "parar» el corazón y mantener al cuerpo oxigenado con la circulación extracorpórea, mientras se realizaba la intervención. 5,6

Desde su advenimiento, la circulación extracorpórea ha permitido llevar a cabo cirugía cardíaca para la corrección de las malformaciones congénitas. Se sabe que su empleo no es inocuo, ya que es capaz de producir, entre otras alteraciones, una respuesta inflamatoria sistémica, que se caracteriza por la activación del complemento, de citocinas, de la coagulación y de fibrinólisis. ${ }^{7}$

A pesar de los avances en las técnicas quirúrgicas y anestésicas la cirugía cardiaca continúa asociada con una importante morbilidad. En este contexto la disfunción renal pos-operatoria es una de las más frecuentes y serias complicaciones con una incidencia reportada en distintas series entre 17 y $72 \%$. La amplitud de este rango puede deberse, entre otros factores, a la variedad de definiciones de IRA utilizadas y a la diversa complejidad de las cirugías incluidas. La disfunción renal post-circulación extracorpórea 
puede aparecer como daño renal subclínico, hasta insuficiencia renal establecida que requiera diálisis en el pos-operatorio; además tiene una patogénesis compleja e involucra mecanismos hemodinámicos e inflamatorios diversos. Aunque se han realizado múltiples estudios, los factores asociados a ésta no están claros en el grupo pediátrico. . $^{-10}$

Estudios previos sugieren que las cirugías cardiacas en la población pediátrica envuelven un riesgo mayor de disfunción y falla renal que en los pacientes adultos debido a los efectos de la cianosis crónica y a la inmadurez de los sistemas orgánicos entre ellos el renal. ${ }^{8}$ El objetivo de la presente investigación fue identificar los factores de riesgo para desarrollar disfunción renal aguda en pacientes pediátricos con cardiopatías congénitas sometidos a corrección quirúrgica bajo circulación extracorpórea, tomando como marcador la alteración del filtrado glomerular calculado por el método de Schwartz y clasificado con la escala de RIFLE adaptada para población pediátrica.

\section{| | | | | | | | | | | | | | | | | | | | | | | | | | | | | | | | | | | | | | | | | | | | | | | | | | | | | | | | | | | | | | | | | | | | | | | | | | | | | | | | | | | | | | | | | | | | | | | | | | | | |}

\section{Material y métodos}

Se realizó un estudio de tipología observacional con diseño ambispectivo y longitudinal, la fase retrospectiva se realizó en los años 2014 y 2015 mientras que la fase prospectiva fue realizada en el año 2016. El tipo de muestreo utilizado fue no probabilístico y consecutivo, los criterios de inclusión fueron pacientes con cardiopatía congénita sometidos a corrección quirúrgica bajo circulación extracorpórea que ingresaron al servicio de terapia intensiva pediátrica durante el periodo de estudio y que presentaron función renal prequirúr- gica normal. El total de cirugías cardiovasculares realizadas durante la investigación fueron 174, de las cuales 96 requirieron circulación extracorpórea, se eliminaron 9 pacientes y finalmente ingresaron a la fase de estudio 87 pacientes, de los cuales recolectamos variables prequirúrgicas, transquirúrgicas y postquirúrgicas. La variable dependiente fue disfunción renal aguda tomando como marcador la alteración del filtrado glomerular calculado por el método de Schwartz y clasificada mediante la escala de RIFLE adaptada a pacientes pediátricos. ${ }^{7,8}$

\section{Tabla I}

Clasificación pRIFLE del daño renal agudo.

\begin{tabular}{|lll|}
\hline Estadios & Aclaramiento de creatinina & Diuresis* \\
\hline Risk (R) & Disminución $25 \%$ & $<0.5 \mathrm{ml} / \mathrm{kg} /$ hora $\times 8$ horas \\
\hline Injury (I) & Disminución $50 \%$ & $<0.5 \mathrm{ml} / \mathrm{kg} /$ hora $\times 16$ horas \\
\hline Failure (F) & Disminución $75 \%$ o $<35 \mathrm{ml} / \mathrm{min} / 1.73 \mathrm{~m}^{2}$ & $<0.3 \mathrm{ml} / \mathrm{kg} /$ hora $\times 24$ horas o anuria $>12$ horas \\
\hline Loss (L) & Insuficiencia renal $>4$ semanas & - \\
\hline End Stage (E) & Insuficiencia renal $>3$ meses & - \\
\hline
\end{tabular}

En recién nacidos $>1.5 \mathrm{ml} / \mathrm{kg} /$ hora.

Modificación pediátrica de la clasificación RIFLE de daño renal agudo. 
Se elaboró una base de datos en el programa SPSS versión 2.1 para analizar la estadística descriptiva de nuestros pacientes utilizando medidas de tendencia central; medidas de dispersión, tablas de frecuencias y porcentajes. Además de análisis univariados y bivariados, se realizó también una base de datos en el programa Minitab versión 17.0 para realizar la estadística inferencial, utilizando regresión lineal múltiple y regresión lineal sim- ple, para buscar el grado de relación entre la variable disfunción renal aguda y las variables independientes pre, trans y postquirúrgicas. También utilizamos la prueba exacta de Fisher para buscar asociación entre las variables categóricas nominales, disfunción renal y muerte, calculando además el índice de riesgo relativo entre dichas variables, considerando significancia estadística un valor de $p<0.05$.

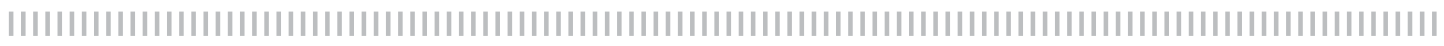

\section{Resultados}

En los 87 pacientes ingresados al estudio, la edad mínima presentada fue de un mes, la máxima de 269 meses (22.5 años) con una media de 36.7 meses. El $54 \%$ de los pacientes fueron menores de 2 años. Las cardiopatías que se presentaron con mayor frecuencia fue la comunicación interventricular en un $31 \%$, seguidas de Tetralogía de Fallot en un $21 \%$, valvulopatías en un $17 \%$, comunicación interauricular en un 16\% y otras cardiopatías en un 15\% (figura 1).

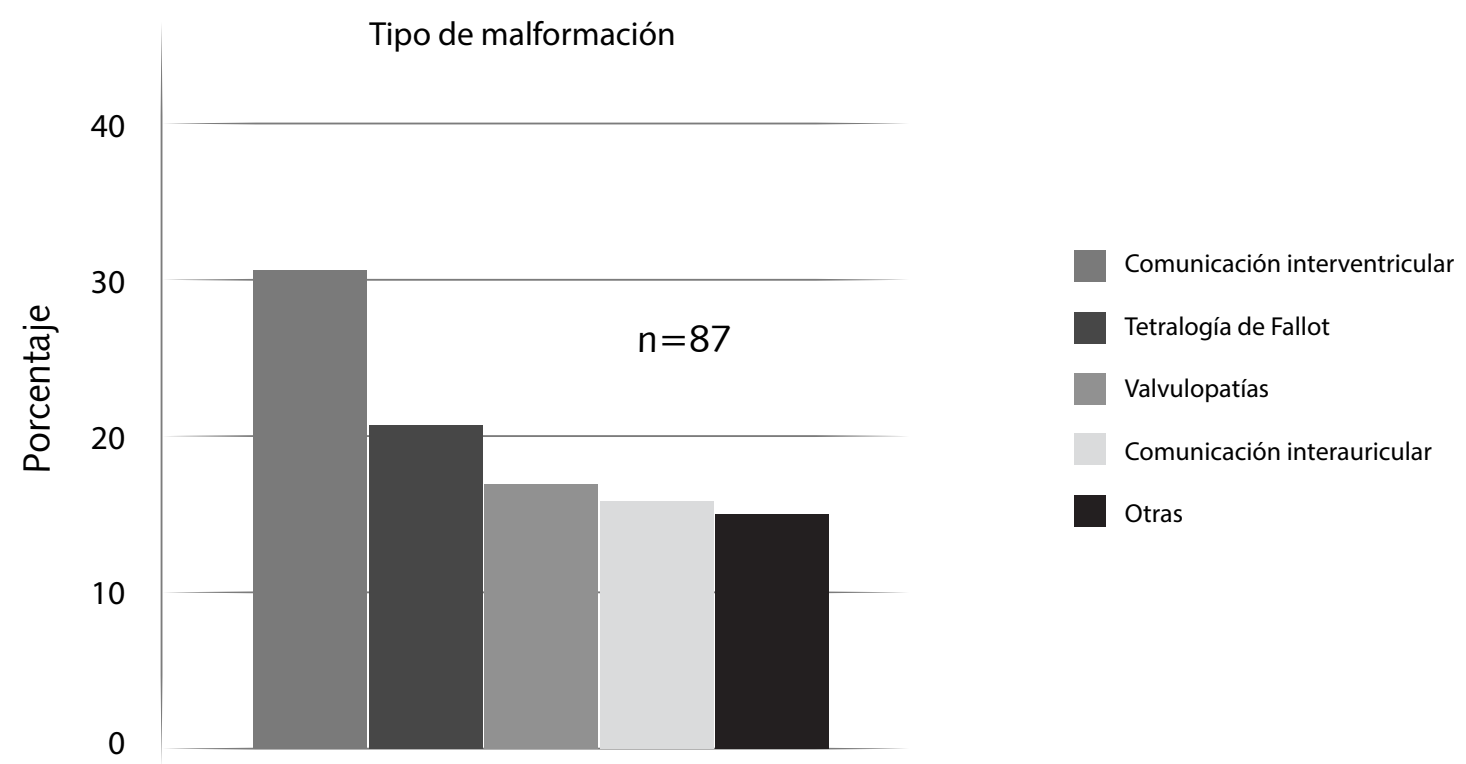

Figura 1. Distribución de los diagnósticos de las malformaciones cardíacas

Observamos que una proporción de los 87 pacientes analizados (con cirugía cardiovascular y circulación extracorpórea) presentó alteraciones en la tasa de filtración glomerular (TFG), clasificándolos con la escala de RIFLE al ingreso del servicio de Unidad de cuidados intensivos pediátricos (UCIP). El 6.9\% (6 pacientes) presentó una Disfunción Renal Aguda (DRA) a las 8h, un $13.8 \%$ (12 pacientes) a las $24 \mathrm{~h}$ y un $23.0 \%$ 
(20 pacientes) a las $48 \mathrm{~h}$. Por su parte, el tiempo de circulación extracorpórea contribuyó significativamente al deterioro del filtrado glomerular ( $p$ 0.000) al igual que tiempo de pinzamiento ( $p$ 0.000), ambos factores afectan negativamente la TFG (figura 2). En nuestro estudio encontramos alta significancia estadística tanto en el tiempo de bombeo como en el tiempo de pinzamiento, encontrando que a mayor tiempo de bombeo y de pinzamiento hay un menor índice en la TFG (figura 2), llegando así a una disfunción renal aguda secundaria.

Cuando analizamos la relación del lactato sérico con el desarrollo de la DRA, ob- servamos que a medida que incrementa el promedio de lactato sérico, también incrementa el porcentaje de pacientes que desarrollaron postquirúrgicamente una DRA. Así, el promedio de lactato sérico postquirúrgico fue de $2.56,3.8$ y $4.39 \mathrm{mmol} / \mathrm{L}$ a las 8,24 y $48 \mathrm{~h}$, respectivamente. De este modo, encontramos una relación estadísticamente significante entre la TFG y la concentración de lactato sérico medido a las 24 y 48 h post-cirugía extracorpórea (figura 3). Por lo tanto, se observó un incremento de la DRA asociada al incremento de los niveles séricos de lactato.
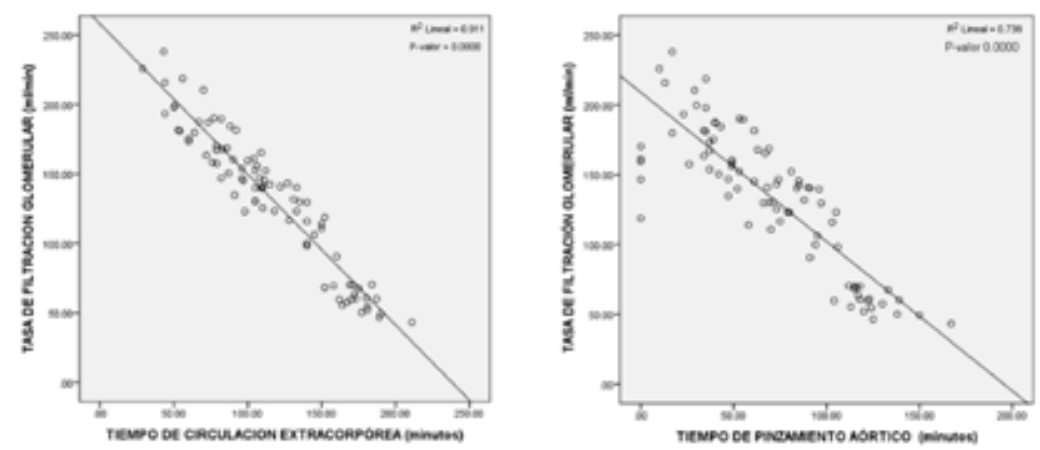

Figura 2: Relación entre la tasa de filtración glomerular (TFG) y el tiempo de circulación extracorpórea (izquierda) o del tiempo de pinzamiento (derecha). El tiempo de bombeo va de 29 a $211 \mathrm{~min}$ y el tiempo de pinzamiento va de 0 a $167 \mathrm{~min}$.

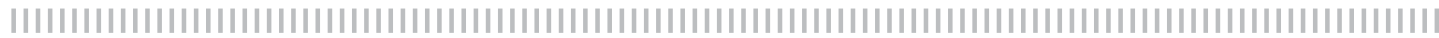
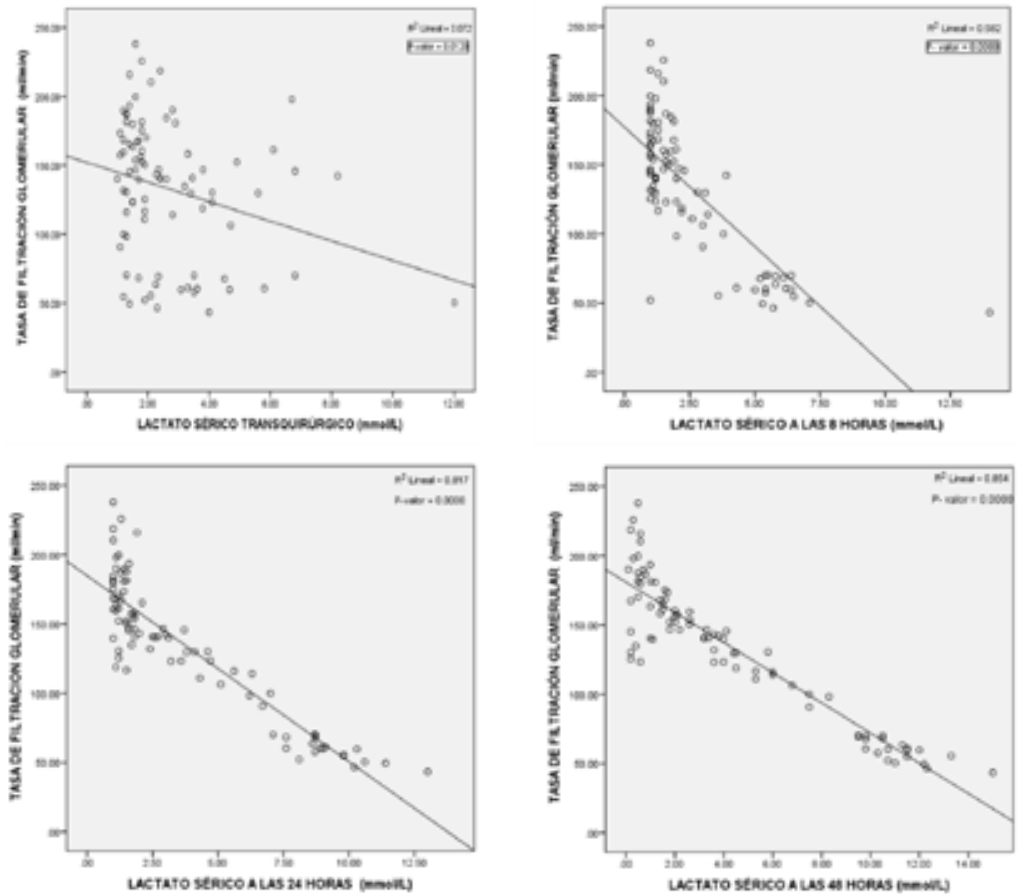

Figura 3: Relación entre la tasa de filtración glomerular (TFG) y los nieles séricos de lactato postquirúrgico a las 0,8 , 24 y $48 \mathrm{~h}$. 
En cuanto al motivo de egreso de los pacientes analizados, el $88.5 \%$ de los pacientes egresaron por mejoría, mientras que el $11.5 \%$ egresaron por defunción. Se observó que todos los pacientes que fallecieron presentaban DRA y se encontró una asociación estadísticamente significante mediante la prueba de Chi cuadrada entre la DRA y defunción. Finalmente, se estimó el riesgo relativo de la DRA en la defunción de este tipo de pacientes y se obtuvo un valor relativo de 5 , con un intervalo de confianza del 95\%. Lo anterior indica que un paciente sometido a circulación extracorpórea que desarrolla DRA, tiene 5 veces más riesgo de morir que uno que no presenta dicha condición.

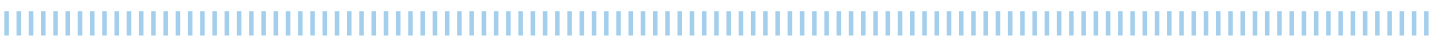

\section{Discusión}

Las cardiopatías congénitas son una causa importante de morbimortalidad a nivel mundial. En nuestro país representan la segunda causa de muerte en menores de 5 años. ${ }^{2}$ Las técnicas para la corrección de las mismas ha avanzado significativamente en los últimos 50 años, siendo la circulación extracorpórea un hito que marcó una revolución en las técnicas de cirugía cardiovascular. ${ }^{3,11}$ Sin embargo, se han reportado múltiples efectos deletéreos secundarios a la técnica de circulación extracorpórea, que promueven el desarrollo de un síndrome de respuesta inflamatoria sistémica.5,7,11 Una de las complicaciones frecuentes en estos pacientes sometidos a corrección quirúrgica bajo circulación extracorpórea es la disfunción renal, la cual si bien puede aparecer de manera subclínica, representa una entidad frecuente con un reporte de incidencia muy variable en la literatura mundial, desde 17 hasta 72\%.8-11 En el Centenario Hospital Miguel Hidalgo, a pesar de ser un centro de referencia que ofrece corrección de cardiopatías congénitas, se cuenta con pocos estudios acerca del impacto de la circulación extracorpórea sobre la función renal, así como de los factores implicados en la disfunción renal postoperatoria. Previamente, se reportó una incidencia de disfunción renal de $12.3 \% .^{12}$

En el presente estudio, el tiempo de circulación extracorpórea mostró una amplia dispersión, con una media de 115.28 mi- nutos, es decir, casi 2 horas, Mientras que el tiempo de pinzamiento reportó una media de 70.97 minutos. La literatura reporta que el síndrome de respuesta inflamatoria comienza a instaurarse desde los primeros minutos de iniciar la circulación extracorpórea, alcanzando un pico 5 horas posteriores al término de la misma. Dado que dicha respuesta inflamatoria es ocasionada por el contacto con las superficies extrañas, la isquemia y la reperfusión, es lógico pensar que la magnitud de la misma sea directamente proporcional a la duración de la circulación extracorpórea y el pinzamiento aórtico.5,11 Observamos que el $32 \%$ de los pacientes presentaron complicaciones postquirúrgicas y una de ellas, tal como lo reporta la bibliografía, fue la DRA, que se presentó en 20 pacientes, es decir, tuvo una frecuencia de $23 \%$, mayor a lo reportado por la doctora Marina Canales en el año $2009^{12}$, pero acorde a los reportes internacionales que la sitúan con un amplio rango entre 17 y $72 \% .{ }^{9}$ De igual forma, nuestro análisis inferencial pudo demostrar una fuerte asociación entre los tiempos prolongados de circulación extracorpórea y pinzamiento aórtico con DRA. Se pudo observar, además, que tiempos prolongados de circulación extracorpórea se asociaron a estancias intrahospitalarias prolongadas, tiempos de ventilación prolongados y tiempos de drenaje torácico prolongados. Esto, a su vez, se asoció a otras complicaciones tales como infeccio- 
nes nosocomiales hasta en el $65 \%$ de los pacientes.

El lactato sérico se ha reportado en la bibliografía como un marcador de hipoxia tisular que podría ser utilizado como predictor de daño renal agudo, lo cual aún es controversial; sin embargo, nosotros encontramos una fuerte asociación entre los niveles DRA y niveles elevados de lactato sérico en el postquirúrgico, específicamente a las $24 \mathrm{y}$ a las 48 h. ${ }^{13}$ Por el momento, herramientas como la escala de RIFLE constituyen una herramienta accesible $y$ fácil de utilizar para la detección de deterioro de la función renal. ${ }^{14-16}$ Es necesario detectar esta complicación de manera temprana e identificar a aquellos pacientes con riesgo de desarrollarla, esto con la finalidad de iniciar las medidas terapéuticas necesarias para evitar alteraciones secundarias como lo son la sobrecarga de volumen y desequilibrio hidroelectrolítico. Dentro de estas medidas se debería de incluir el inicio temprano de terapia sustitutiva renal.

\section{Conclusiones}

La disfunción renal secundaria es una complicación frecuente de la cirugía cardiovascular con circulación extracorpórea y condiciona un incremento importante de la morbimortalidad en las unidades de cuidados intensivos pediátricos en todo el mundo. En nuestro estudio, a través de prueba exacta de Fisher se observó una asociación significativa entre DRA y la muerte de los pacientes, con un riesgo relativo encontrado de 5. La DRA, por lo tanto, incrementa el riesgo de morir hasta 5 veces en comparación con aquellos que no la presentan. La escala de RIFLE puede ser una escala útil para el diagnóstico temprano de disfunción renal aguda.

\section{Bibliografía}

1. Cervantes, Jorge et al. El Registro Mexicano de Cirugía Cardiaca Pediátrica. Primer informe. Evidencia Médica e Investigación en Salud. [Revista en línea]. 2014. [citado 2017 Ene 12]; 7 (12). Disponible en: http://www.medigraphic.com/pdfs/revinvcli/nn2013/nn136c.pdf

2. Varela, Javier et al. Epidemiología de pacientes con cardiopatías congénitas sometidos a cirugía en un hospital privado de tercer nivel en México. Revista de Investigación Médica Sur. [Revista en línea]. 2015. [citado 2017 Ene 12]; 22 (4). Disponible en: http:// medicasur.org.mx

3. Instituto Nacional de Estadística y Geografía. (2015). Defunciones de menores de un año, México. Disponible en: http://www.inegi.org.mx

4. Archivo clínico del Centenario Hospital Miguel Hidalgo. Enero, 2017.

5. Sanz, Marcano et al. Cirugía cardiovascular pediátrica en normotermia durante la circulación extracorpórea. Revista Electrónica de las Ciencias Médicas en Cienfuegos. [Revista en línea]. 2012. [citado 2017 Ene 12]; 10 (1). Disponible en: http://www.redalyc.org/ articulo.oa?id $=180023041007$
6. Brotons, Albert et al. El desarrollo de la cardiología pediátrica española y su impacto en el manejo de las cardiopatías congénitas. An Pediatr (Barc). [Revista en línea]. 2015. [citado 2017 Ene 12]; 83 (5). Disponible en: http://www.analesdepediatria.org/en/thedevelopment-spanish-paediatric cardiology/articulo/ S2341287915001982

7. Curi-Curi, Pedro et al. Impacto intraoperatorio de la ultrafiltración modificada en pacientes pediátricos sometidos a cirugía cardíaca con circulación extracorpórea. Cirugía Cardiovascular. [Revista en línea]. 2016. [citado 2017 Ene 10]; 23 (4). Disponible en: https:// doi.org/10.1016/j.circv.2016.02.003

8. Cabas, Lilian et al. Disfunción renal en postoperatorio de cirugía cardíaca pediátrica con circulación extracorpórea. Revista Colombiana de Anestesiología. [Revista en línea]. 2005. [citado 2017 Ene 12]; 33 (2). Disponible en: http://www.redalyc.org/articulo. oa?id=195114536002

9. Ovalle B, Pilar et al. Reemplazo renal en el postoperatorio de niños sometidos a cirugía cardíaca con circulación extracorpórea. Rev. chil. pediatr. [Internet]. 2012 [citado 2017 Ene 12]; 83( 1 ): 24-32. Disponible en: http://dx.doi.org/10.4067/S037041062012000100003. 
10. Facenda, Abel et al. Efectos de la circulación extracorpórea sobre el filtrado glomerular en la cirugía cardiovascular pediátrica. Rev. Colom. Cardiol. [Internet]. 2011. [cited 2017 Jan 12]; 18 (3). Available from: http://www.scielo.org.com/ scielo.php?script=sci arttext\&pid $=$ S0120-56332011000300007\&lng $=$ en .

11. Precis d'anesthesie cardiac. [Página principal de internet]. Lausanne, 2010. [Actualizado Enero 2015]. http://www.precisdanesthesiecardiaque.ch/index. html.

12. Canales, Marina. Incidencia de insuficiencia renal asociada a cirugías de cardiopatías congénitas. Factores asociados y evolución. [Tesis]. Aguascalientes: Universidad Autónoma de Aguascalientes. Facultad de Medicina; 2009.

13. Ruan, Caixia, Shang, Wei, Liu Yazhou et al. Postoperative hyperlactatemia is linked to acute kidney injury after type $\mathrm{A}$ aortic dissection surgery: a retrospective study. International Journal of Clinical and Experimen- tal Medicine. [Revista on-line] 2016 [Consultado 24 enero 2017]; 9(7). Available from: http://www.ijcem. com/files/ijcem0027697.pdf

14. Aspelin, Peter, Barsoum, Rashad, Buedmann, Emmanuel et al. Kidney disease: Improving global outcomes (KDIGO) CKD work group. KDIGO Clinical Practice Guideline for Acute Kidney Injury. Kidney international Supplements. 2012; 2: 1-138. Available from: http://kdigo.org/home/guidelines/acute-kidney-injury/

15. Salgado, Landa, Masevicius, Gianassi, San-Román, Silva, et al. Insuficiencia renal aguda según RIFLE y AKIN: estudio multicéntrico. Med Intensiva. 2014; 38: 271-277.

16. Asociación Española de Pediatría [Página principal de internet]. Madrid: Protocolos de nefrología; c2014 [consultada 15 de Julio 2016][aprox. 3 pantallas]. Disponible en: http://www.aeped.es/sites/default/files/ documentos/22_dano_renal_agudo.pdf 